\title{
The effects of cannabis on memory function in users with and without a psychotic disorder: findings from a combined meta-analysis
}

\author{
T. Schoeler ${ }^{1}$, J. Kambeitz ${ }^{1,2}$, I. Behlke ${ }^{1,3}$, R. Murray $^{1}$ and S. Bhattacharyya ${ }^{1 *}$ \\ ${ }^{1}$ Department of Psychosis Studies, Institute of Psychiatry, Psychology \& Neuroscience, King's College London, De Crespigny Park, London SE5 8AF, UK \\ ${ }^{2}$ Department of Psychiatry, Ludwig-Maximilian-University Munich, Nußbaumstr. 7, 80336 Munich, Germany \\ ${ }^{3}$ Institute of Psychology, Univeristy of Osnabrueck, Seminarstr. 20, 49074 Osnabrueck, Germany
}

Background. Effect of cannabis use on memory function is a contentious issue, with effects being different in healthy individuals and patients with psychosis.

\begin{abstract}
Method. Employing a meta-analytic approach we investigated the effects of cannabis use on memory function in patients with psychosis and healthy individuals, and the effect of diagnosis, memory dimension and moderating factors. A total of 88 studies were identified through a systematic literature search, investigating healthy $(n=7697)$ and psychotic $(n=3261)$ individuals. Standardized mean differences between the cannabis user and non-user groups on memory tasks were estimated using random-effects models and the effect-size statistic Cohen's $d$. Effects of potential moderating factors were tested using mixed-effects models and subgroup analyses.
\end{abstract}

Results. We found that cannabis use was associated with significantly $(p \leqslant 0.05)$ impaired global $(d=0.27)$ and prospective memory $(d=0.61)$, verbal immediate $(d=0.40)$ and delayed $(d=0.36)$ recall as well as visual recognition $(d=0.41)$ in healthy individuals, but a better global memory $(d=-0.11)$, visual immediate recall $(d=-0.73)$ and recognition $(d=-0.42)$ in patients. Lower depression scores and younger age appeared to attenuate the effects of cannabis on memory. Cannabis-using patients had lower levels of depression and were younger compared with non-using patients, whilst healthy cannabis-users had higher depression scores than age-matched non-users. Longer duration of abstinence from cannabis reduced the effects on memory in healthy and patient users.

Conclusions. These results suggest that cannabis use is associated with a significant domain-specific impairment in memory in healthy individuals but not in cannabis-using patients, suggesting that they may represent a less developmentally impaired subgroup of psychotic patients.

Received 3 September 2014; Revised 29 July 2015; Accepted 4 August 2015; First published online 10 September 2015

Key words: Cannabis, memory, meta-analyses, psychosis, $\Delta$-9-tetrahydrocannabinol.

\section{Introduction}

Cannabis is one of the most frequently used illicit drugs in the world (UN Office on Drugs and Crime, 2010). Young people are using it at an earlier age (Smith \& Flatley, 2011), to the extent that it is replacing cigarette smoking as the most common substance used by them (Moss et al. 2013). Based on evidence from observational studies, cannabis use has been linked to a number of effects on cognition and behaviour (Ranganathan \& D'Souza, 2006; Solowij \& Pesa, 2010), with memory being one of the most robustly replicated cognitive functions which has been reported to be impaired following chronic (non-acute) cannabis

* Address for correspondence: S. Bhattacharyya, M.B.B.S., M.D., Ph.D., Department of Psychosis Studies, Institute of Psychiatry, King's College London, De Crespigny Park, London SE5 8AF, UK.

(Email: sagnik.2.bhattacharyya@kcl.ac.uk) use (Grant et al. 2003; Fletcher \& Honey, 2006; Solowij \& Battisti, 2008; Solowij \& Pesa, 2010; Schoeler \& Bhattacharyya, 2013). Meta-analyses of observational studies comparing cannabis-using subjects with nonusing subjects have reported small (Grant et al. 2003) to medium-sized effects (Schreiner \& Dunn, 2012) of cannabis on verbal memory performance, consistent with evidence that regular cannabis use affects the structure (Matochik et al. 2005; Yucel et al. 2008) and function (Kanayama et al. 2004; Sneider et al. 2008) of brain regions involved in memory processing. As discussed by Solowij \& Battisti (2008), accumulating evidence suggests that the magnitude and persistence of cognitive impairment associated with cannabis use depends on various parameters such as age of onset (Pope et al. 2003; Gruber et al. 2012), dose (Bolla et al. 2002), frequency (Jager et al. 2006; Tait et al. 2011), and duration of cannabis use (Meier et al. 2012) as well as the period of abstinence from cannabis (Pope 
et al. 2001) at the time of testing, all of them interacting with each other to predict the degree of impairment (Wagner et al. 2010).

$\Delta$-9-Tetrahydrocannabinol (THC), the main psychoactive ingredient of cannabis, is thought to be responsible for the adverse effects of cannabis on cognition. Experimental studies involving acute challenge with THC have reported that cannabis can impair shortterm (Morrison et al. 2009; Englund et al. 2013) and long-term memory function (Curran et al. 2002; D'Souza et al. 2004) and alter the neural substrates underlying learning and memory (Bhattacharyya et al. 2009, 2012) in healthy subjects, for which reason THC administration has also been proposed as a strategy to model and study schizophrenia pathophysiology (Fletcher \& Honey, 2006).

Among individuals suffering from a psychotic disorder, co-morbid substance use disorders including cannabis use are higher than in the general population (Koskinen et al. 2010). Cannabis use is also associated with an increased risk of relapse (Linszen et al. 1997), and of violence and criminal activity (Miles et al. 2003) in patients with psychosis. Although experimental acute challenge studies suggest that acute impairments in memory induced by THC are greater in those with a psychotic disorder (D'Souza et al. 2005) than healthy controls, evidence from naturalistic studies in patients with psychosis have not been as straightforward. Epidemiological studies and meta-analyses investigating the non-acute effects of cannabis use in patient samples have either reported better performance in different domains of memory such as working memory (Jockers-Scherubl et al. 2007; Løberg et al. 2008; Rabin et al. 2011) and verbal learning (Schnell et al. 2009; Leeson et al. 2012) or no significant difference between cannabis-using and non-using patients (Korver et al. 2010; Donoghue et al. 2012; Bahorik et al. 2013).

The evidence summarized above suggests that the same drug, which is known to be a risk factor for psychosis and is associated with memory impairment in healthy users, appears to have an association with better memory performance in users with a psychotic disorder. In light of these paradoxical trends in the non-acute effects of cannabis use on memory in healthy users $v$. those with psychosis, especially when this is contrasted with experimental evidence of the acute effects in these two groups (D'Souza et al. 2005), the objective of the present study was to systematically summarize the non-acute effects (based on observational studies) of cannabis use on memory function both in those with and without a psychotic disorder. Elucidating this is important to advance current understanding of the association between cannabis use and psychosis and determinants of this association, which in turn may help devise intervention strategies. To our knowledge, no study has as yet carried out a systematic quantitative synthesis of the literature employing meta-analytic techniques in both healthy and psychotic users regarding the long-term effects of cannabis use on the different subdomains within memory function, defined as a multidimensional construct.

We employed a meta-analytic approach to statistically integrate the results from multiple individual studies. In the present analysis we have focused on non-acute effects, thus including both residual and chronic effects. We employed an inclusive definition in order to have the power to detect the effect of cannabis use in both healthy and psychotic users. Hence, all studies comparing cannabis users with non-users were included, independent of the time of abstinence from cannabis/results from urine tests. However, to address the issue of residual effects, we examined the effect of abstinence by carrying out moderation analysis (described below). This allowed us to address the following questions:

(i) Does the non-acute effect of cannabis on memory function differ significantly between healthy individuals and those with a psychotic disorder?

(ii) Is there a domain-specific effect of cannabis on memory?

(iii) Are there particular demographic and individual differences between healthy cannabis users and cannabis-using patients with psychosis that may be related to the differential effects of cannabis use on memory in these two groups of cannabis users?

(iv) Does the magnitude of the effect of cannabis use on memory depend on certain cannabis use characteristics such as frequency, duration, age of onset of use and duration of abstinence at the time of testing?

\section{Method}

\section{Search strategy and selection criteria}

Following recommended guidelines (Higgins \& Green, 2008; Beller et al. 2013), a systematic search strategy was used to identify all relevant studies. The MEDLINE database was searched for English language studies using a combination of search terms describing the outcome of interest (neuropsych*, cognit*, memory, learning, recall) and cannabis use (marijuana/ marihuana, cannabis, THC, cannabinol, cannabidiol), with the final search conducted on 27 June 2014. Following further bibliography searches of previously published reviews and meta-analyses (Gonzalez et al. 
2002; Grant et al. 2003; Potvin et al. 2008; Rabin et al. 2011; Donoghue \& Doody, 2012; Schreiner \& Dunn, 2012; Yücel et al. 2012), studies were selected if they met the inclusion criteria (for a flowchart, see Supplementary Fig. S1).

\section{Recorded variables}

Memory was considered as a multidimensional construct (Kambeitz et al. 2012), including 11 distinct memory dimensions, classified based on temporal characteristics (i.e. from short-term memory to longterm memory) or based on its content (i.e. verbal or visual memory) (Pezdek \& Evans, 1979) (see Supplementary Fig. S1 and Supplementary Table S1). As a common metric for the estimate of effect size, Cohen's $d$ or the standardized mean difference (SMD) between the means of cannabis users $v$. non-users was estimated for each neuropsychological test that measured an aspect of memory functioning. This was followed by sensitivity analysis in a more homogeneous group (Kambeitz et al. 2012) that involved computing the effect-size estimate using the same approach as above, but including only the data from studies that employed the same memory test (see Supplementary Appendix S1). Demographic and clinical characteristics [pre-morbid intelligence quotient (IQ), depression, anxiety and level of global functioning] were compared using another set of SMDs estimated from data for those measures reported as means and standard deviations (see Supplementary Table S2).

Following established guidelines, subgroup and metaregression analyses were carried out for duration, frequency, age of onset and abstinence from cannabis use as well as for cigarette and alcohol use and year of publication (for classification criteria, see Supplementary Appendix S1).

\section{Analysis}

Analyses were conducted with the metaphor package (Viechtbauer, 2010) in R, using random-effects models (Lane et al. 2005) that assume that the SMDs vary from study to study (Borenstein et al. 2011) (for details, see Supplementary Appendix S1). Cohen's $d$ for memory outcome was estimated according to established guidelines (Higgins \& Green, 2008). In a second set of random-effects models, SMDs for demographic and clinical variables in cannabis users $v$. non-users were estimated. Missing data, potential publication bias and heterogeneity assessment were considered following recommended procedures. To investigate sources of heterogeneity, the influence of continuous moderator variables, including the SMDs estimated for demographic and clinical variables, on Cohen's $d$ for memory was tested using mixed-effect models (Viechtbauer, 2010).

\section{Results}

After excluding irrelevant studies or those that did not satisfy our criteria, a final sample of $n=88$ studies (number of domain-specific substudies $k=303$ ), comprising 7697 healthy subjects and 3261 subjects with a psychotic disorder was included (see Supplementary Fig. S1 and Supplementary Table S3).

\section{Random-effects models: effect of cannabis on memory function in cannabis users with and without a psychotic disorder}

Cannabis use significantly impaired global memory $(d=0.27, p<0.0001$; Fig. 1$)$ in healthy cannabis users relative to non-users. Conversely, in patients with psychosis, cannabis use was associated with better performance in these tasks, with a modest effect size $(d=-0.11, p=0.05)$. Substantial overall heterogeneities $\left(I^{2}=72 \%\right.$, see Supplementary Table S4) indicated that moderators are likely to have influenced the effect of cannabis use on memory (Song et al. 2001). Moderator analysis indicated that the effect-size estimates were significantly different $(p<0.0001)$ between the two diagnostic groups (patient $v$. healthy).

In the healthy sample, most of the memory dimensions were significantly impaired by cannabis use, with the most pronounced impairments being observed for prospective memory. Within the patient sample, cannabis users had either no impairments or performed significantly better in several assessed memory dimensions (working memory, visual immediate recall, visual recognition, verbal recognition) (Fig. 1). Moderator analysis indicated that the effectsize estimates were significantly different $(p<0.001)$ between the different memory dimensions within the healthy sample, but not in the patients $(p=0.15)$. Dimension-specific effects in healthy individuals were not related to the size of sample examined for each dimension (Supplementary Table S5). Funnel plots (Supplementary Fig. S2) and Egger's test indicated that publication bias was present within the healthy sample $(p<0.005)$ but not the patient sample $(p=$ $0.34)$ for global memory. The corrected model revealed an effect-size estimate of Cohen's $d=0.18(p<0.0001)$. Supplementary Table S6 details the test results for publication bias for global memory and each memory dimension separately.

Sensitivity analysis to address the issue of heterogeneity in the measures employed resulted in an even larger-sized, statistically significant effect of cannabis use on immediate recall $(d=0.52, p<0.0001)$ 


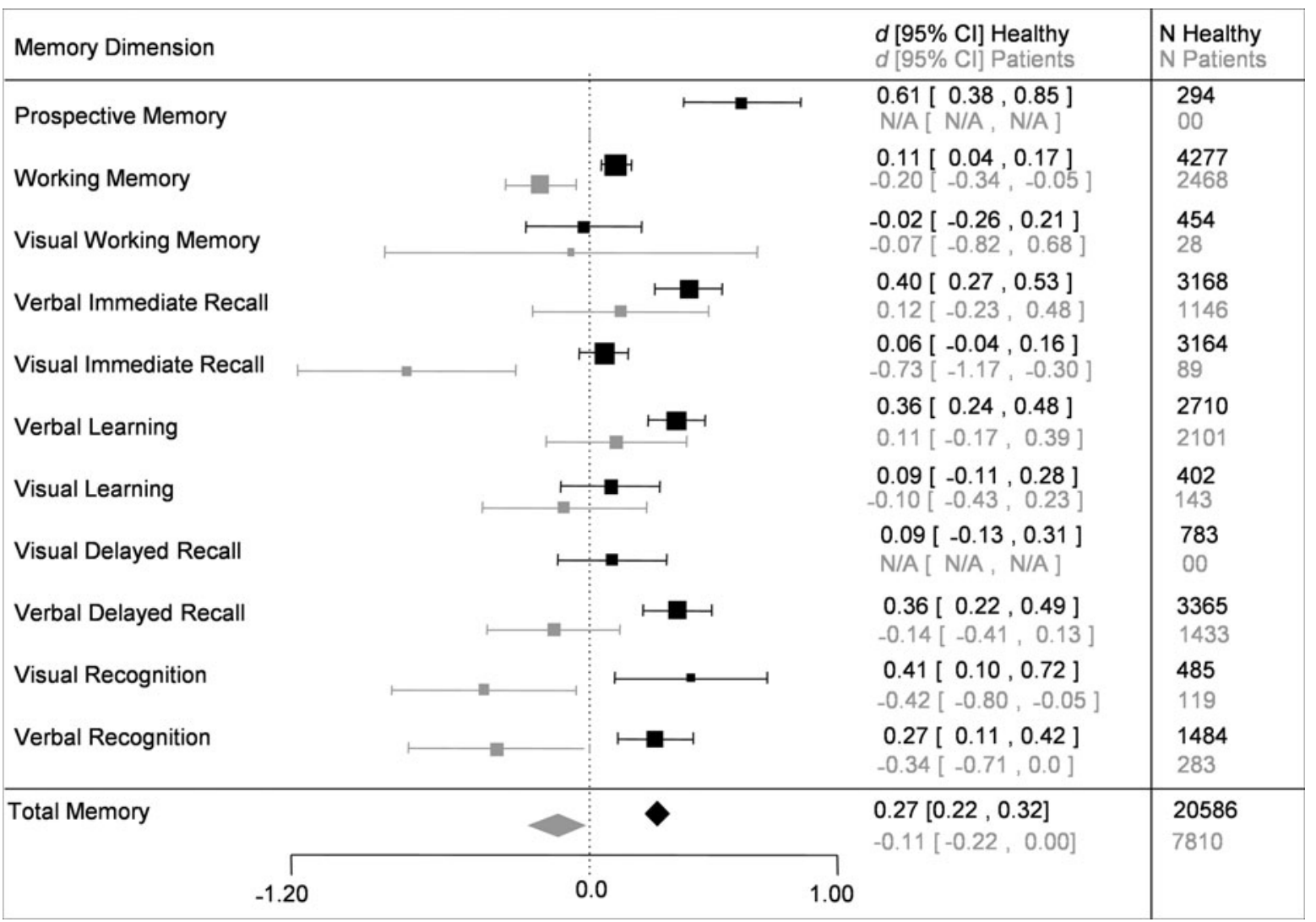

Fig. 1. Random-effects models for global memory and different memory dimensions in healthy subjects ( $\square$ ) and patients ( $\square$ ). Effect-size estimate Cohen's $d$ indicates differences in memory outcome between non-users and cannabis users, with larger estimates reflecting worse performance in cannabis users. $N$ is the number of subjects per memory dimension, including cannabis users plus non-users. CI, Confidence interval.

and verbal learning $(d=0.52, p<0.0001)$ in the healthy sample by including only studies that employed the Rey Auditory Verbal Learning Test (RAVLT) (see Supplementary Table S7). Results with the RAVLT in the patient sample were similar (immediate recall: $d=-0.10$; verbal learning: $d=-0.05$ ) to the larger inclusive sample.

\section{Mixed-effects models: test of moderators \\ Clinical moderators of effects of cannabis action}

Meta-regression (Supplementary Table S8) suggested a significant moderating effect of SMDs (SMDs between cannabis users and non-users) estimated for level of functioning, pre-morbid IQ and level of depression, implicating a more pronounced effect on global memory in those cannabis-using subjects with a lower level of functioning and pre-morbid IQ, as well as higher levels of depression relative to non-using controls. We then examined the extent to which these factors may have contributed to the different patterns of effect of cannabis in healthy and patient groups, by comparing the SMDs estimated for each moderator variable per study between the two diagnostic groups (healthy v. patients). As shown in Fig. 2, healthy cannabis users showed higher levels of depression when compared with non-users (SMD $=-0.44, p<0.0001)$, while in the patient sample cannabis users had lower levels of depression than non-users $(\mathrm{SMD}=0.27, p<0.0001)$. Healthy cannabis users were characterized by lower levels of functioning when compared with healthy non-users $(\mathrm{SMD}=0.83, p=0.002)$, whilst no such effect was present in the patient sample $(\mathrm{SMD}=0.06, p=$ 0.43).

\section{Effect of age}

Meta-regression showed that age at the time of performance of memory tasks significantly moderated the adverse effects of cannabis use on memory (slope coefficient of $-0.20, p<0.0001)$. This effect was such that the younger the cannabis user compared with the non-user at the time of cognitive testing, the smaller was the magnitude of the adverse effect of cannabis use on memory function. While cannabis users in the healthy sample appeared to be matched in terms of 


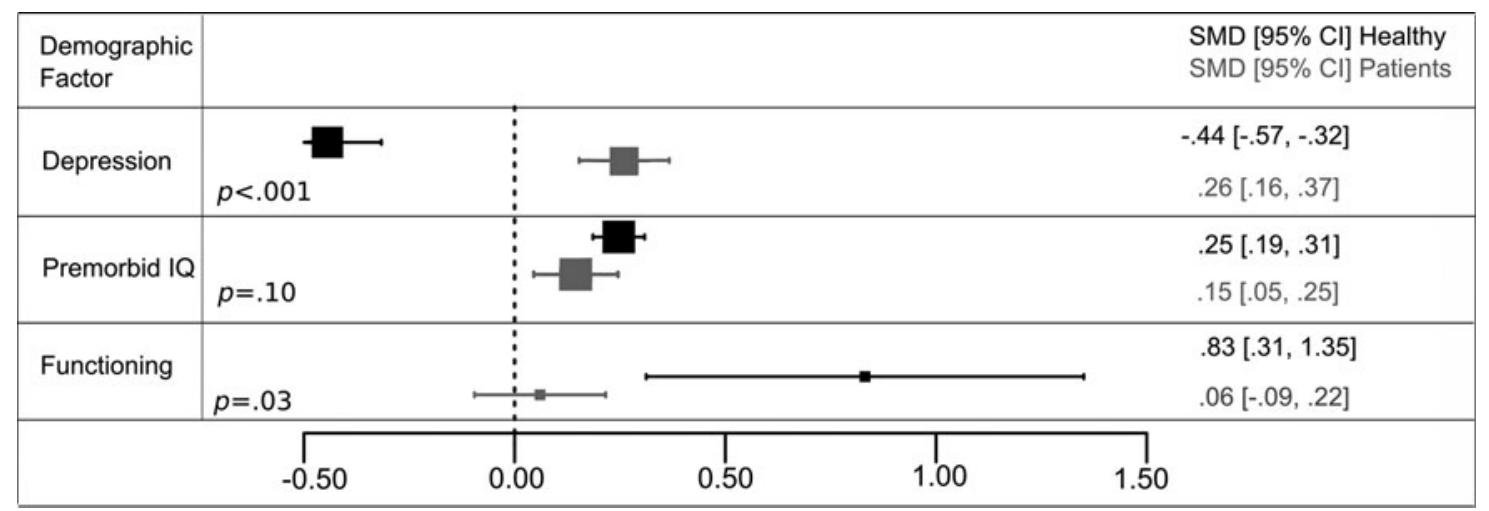

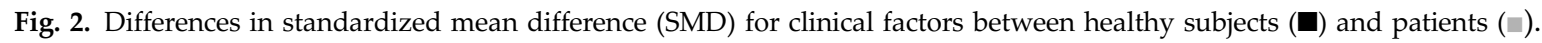
SMDs in clinical parameters [depression scores, pre-morbid intelligence quotient (IQ) and functioning scores] are shown between non-users and cannabis users, with larger SMDs reflecting lower scores (lower depression levels, lower pre-morbid IQ, lower functioning) in cannabis users. The $p$ value is the estimated significance level for the categorical moderator (healthy $v$. patients) in mixed-effects models for SMD in depression scores, pre-morbid IQ and functioning scores between non-users and cannabis users. CI, Confidence interval.

their mean age to the non-users (25.6 years $v .25 .6$ years, $t=0.30, p=0.76$ ), the cannabis-using patients were on average 5 years younger than the non-using patients $($ mean $=32.5$ years $v .27 .6$ years, $t=9.08, p<$ 0.0001) (see Fig. 3).

\section{Effect of cannabis use characteristics}

Categorical moderator analysis revealed that those cannabis users in the healthy and patient groups abstaining from the substance for 10 days or more before performing memory tasks did better in memory tests when compared with those who had abstained for less than 10 days (Fig. 4). In the healthy sample, significantly greater memory impairments were present in heavy users when compared with moderate or light users, while the frequency of cannabis use was not a significant moderator in the patient sample. There was a trend toward a dose-response relationship $(p=0.06)$ between the duration of cannabis use and effect on memory, such that larger effect sizes were reported in long-term healthy cannabis users compared with short-term users. Age of onset of cannabis use did not appear to moderate the adverse effects of cannabis in both groups.

\section{Effect of difference in co-morbid alcohol and nicotine use}

Subgroup analysis suggested that differences in alcohol and nicotine use patterns did not significantly $(p<0.05)$ moderate the effects of cannabis on memory function (see Supplementary Fig. S3).

\section{Effect of year of publication}

In the healthy sample, there was a significant effect of date of publication on the effect of cannabis on memory, i.e. studies that were published before 2000 reported a significant but small effect size of $d=0.12$, whereas studies published after 2000 reported a significant moderate effect size of $d=0.31$, which were significantly different in comparison ( $p=0.01$; see Fig. 5$)$. This comparison could not be carried out in the patient sample, considering that all of the studies in patients were published after 2000 (see Supplementary Table S3).

\section{Discussion}

Results presented here suggest that cannabis use has a robust but modest adverse effect on global memory function as well as its multiple component dimensions in healthy cannabis users, with the precise magnitude of the effect depending on the specific dimension of memory tested. This replicates the results of previous reviews (Solowij \& Battisti, 2008) and meta-analyses that reported small impairments in memory in healthy cannabis users, with effect sizes ranging from $d=0.16$ to $d=0.32$ (Grant et al. 2003; Schreiner \& Dunn, 2012). Furthermore, our results implicate selective effects of cannabis on aspects of memory in healthy users, with the largest effect sizes reported for prospective $(d=$ 0.61 ) and retrospective memory with both verbal (e.g. verbal learning, immediate and delayed recall) and visual content (e.g. visual recognition). In practical terms, these results suggest that the effects of cannabis use are more likely to be expressed in impairments in day-to-day activities that require the ability to remember to carry out the intended task at some point of time in the future (e.g. meeting a friend or taking medication) or problems in recalling events from the past (e.g. recalling past conversations). The effects on rather 


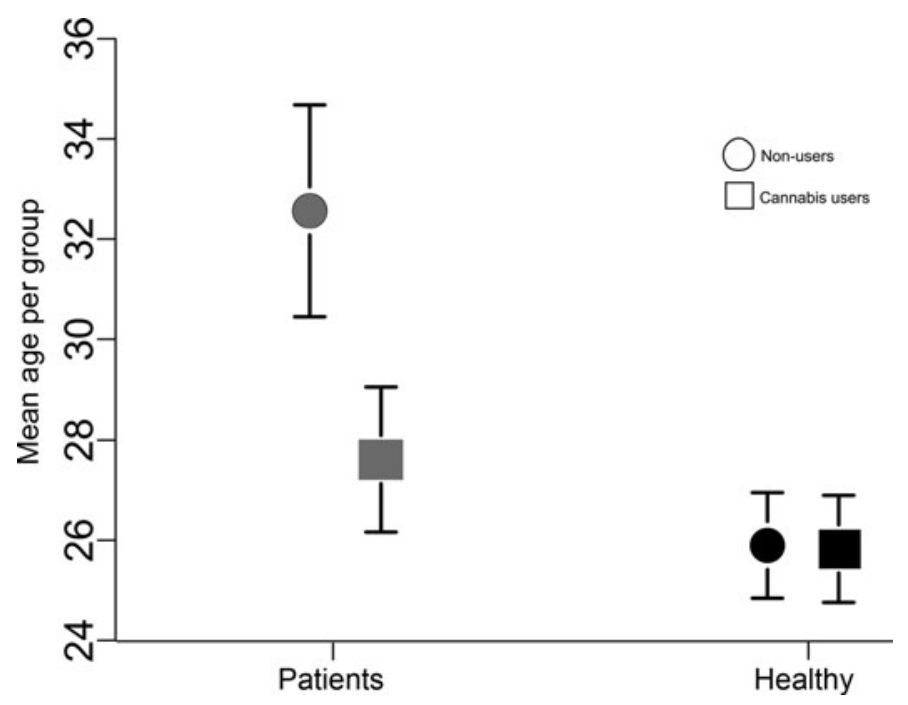

Fig. 3. Mean differences in age between groups. There were significant differences in mean age between cannabis users and non-users in patients $(p<0.0001)$ but not the healthy sample $(p=0.76)$.

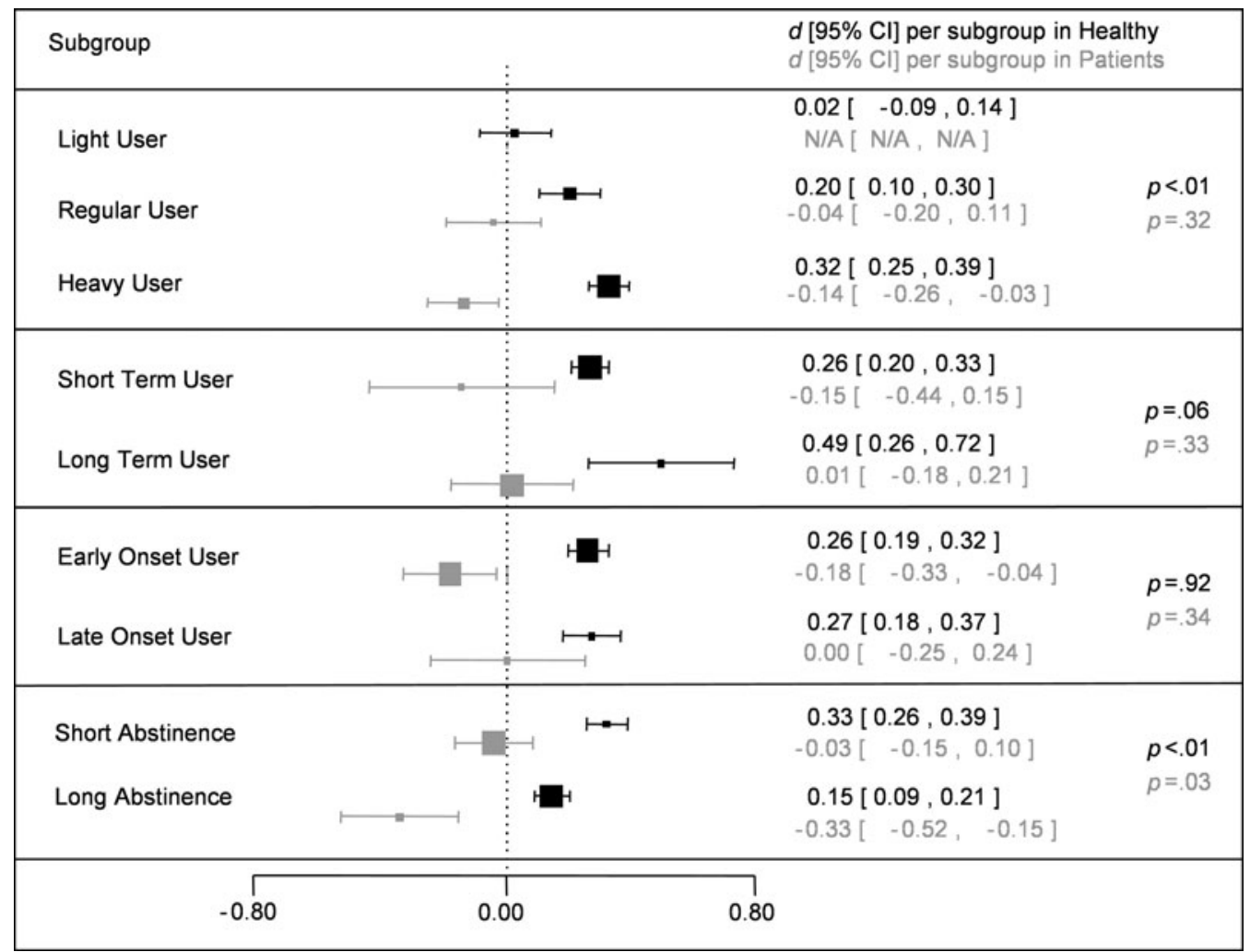

Fig. 4. Categorical moderator analysis based on cannabis use patterns in healthy subjects $(\square)$ and patients ( $\square$ ). Effect-size estimate Cohen's $d$ indicates differences in memory outcome between non-users and cannabis users, with larger estimates reflecting worse performance in cannabis users. The $p$ value is the estimated significance level for each categorical moderator (i.e. frequency of use, duration of use, onset of use, abstinence from cannabis) in moderator analysis. 'Light user' indicates less than four times/month or less than 10 joints/month; 'regular user' indicates between four and 20 times/month or between 10 and 25 joints/month; 'heavy user' indicates diagnosis of cannabis dependence or more than 20 times/month or more than 30 joints/month; 'short term user' indicates cannabis use less than 2500 days of use; 'long term user' indicates more than 5000 days of use; 'early onset user' indicates cannabis use before the age of 17 years; 'late onset user' indicates cannabis use after the age of 17 years; 'short abstinence' indicates less than 10 days of abstinence; 'long abstinence' indicates more than 10 days of abstinence; CI, confidence interval; N/A, not applicable. 


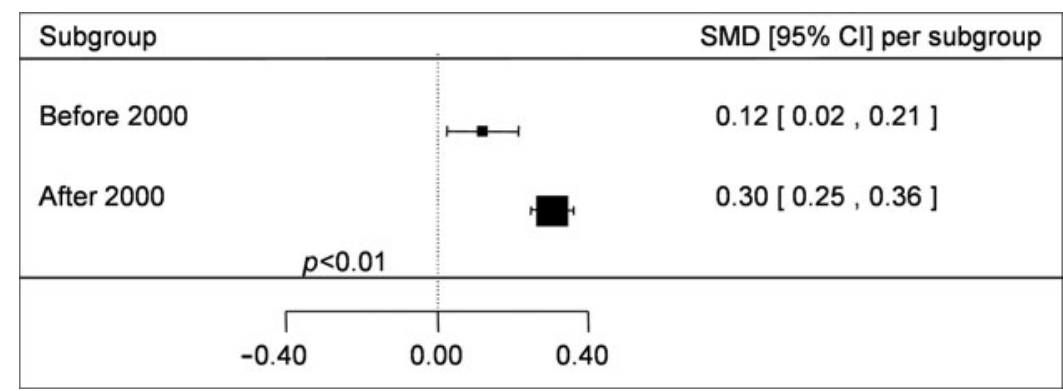

Fig. 5. Categorical moderator analysis: year of publication. Standardized mean differences (SMDs) are shown in memory outcome between non-users and cannabis users in the healthy sample, with larger SMDs reflecting worse performance in cannabis users. The $p$ value is the estimated significance level for the categorical moderator year of publication (before $2000 v$. after 2000). 'Before 2000' indicates studies published before the year 2000; 'after 2000' indicates studies published after the year 2000. CI, Confidence interval.

longer-term memory functions may also reflect the high density of $\mathrm{CB} 1$ receptors, the main target of cannabinoids, in brain structures critically involved in longer-term memory processes such as the hippocampus, basal ganglia and cerebellum (Herkenham et al. 1990; Eichenbaum, 1993; Packard \& Knowlton, 2002), with cannabinoids preventing hippocampal long-term potentiation, a potential substrate for memory (for a review, see Iversen, 2003) through a downstream effect on a number of neuromodulator/ neurotransmitter systems (Pertwee, 2008). Strikingly, the results indicate better overall memory performance in cannabis-using patients when compared with non-users $(d=-0.11)$, particularly for the domains of visual immediate recall $(d=-0.73)$, verbal recognition $(d=-0.34)$ and visual recognition $(d=-0.41)$, which is in line with earlier meta-analytical findings of either a lack of adverse effect of cannabis on cognitive abilities in patients with a psychotic disorder (Løberg \& Hugdahl, 2009; Yücel et al. 2012) or association with better attention and psychomotor speed (Donoghue \& Doody, 2012), reasoning, problem-solving and visual memory (Potvin et al. 2008) and neurological soft signs (Ruiz-Veguilla et al. 2012). A recent meta-analysis also reported that psychotic patients with unspecified co-morbid substance use disorder performed significantly better in tests measuring verbal learning and memory $(d=-0.26)$ than those without (Donoghue \& Doody, 2012). Better verbal memory function related to substance use has also been reported in a subsequent study comprising a sample of first-episode psychosis for verbal memory function (Lutgens et al. 2014), while no effect of polydrug use was present on working memory in schizophrenia patients (Wojtalik \& Barch, 2014). Similarly, no adverse effects of cocaine on verbal learning and memory were present in patient samples (Donoghue \& Doody, 2012). In summary, cannabis appears to have differential effects on memory in users with and without a psychotic disorder, a phenomenon that has also been referred to as the paradox effect of the dually diagnosed (Penk et al. 2000; Moreno-Granados et al. 2014).

Meta-regression indicated that several factors such as lower levels of depression, higher levels of functioning and a younger age at the time of cognitive assessment may have attenuated the adverse effects of cannabis on memory, whilst other factors such as years of education, level of anxiety, co-morbid nicotine and alcohol use did not appear to moderate the effects of cannabis. First, on comparing the healthy and patient groups, cannabis-using patients appeared to be less depressed than the non-users, while an opposite pattern was evident in the healthy sample. This may suggest that co-morbid depressive symptoms may partly account for the apparent opposite effects of cannabis use on memory function in healthy users $v$. those with a psychotic disorder. In fact, depressive symptoms have been associated with impairments in cognitive function in healthy subjects, including episodic and semantic memory, as well as executive function (McDermott \& Ebmeier, 2009), and have also been linked to impairments in recall and recognition memory in schizophrenia patients (Burt et al. 1995).

Second, age differences between cannabis users and non-users may have significantly moderated the effect of cannabis use on memory. Adverse effects of cannabis on memory seemed to increase with increasing age differences between cannabis users and non-user (i.e. the older the cannabis user in comparison with the non-user, the larger the impairing effect of cannabis on memory), consistent with a previous meta-analysis reporting an association between older age and a stronger detrimental effect of cannabis use on global cognition, as well as on working memory and processing speed in patients with a psychotic disorder (Potvin et al. 2008). This may in fact be an effect of age-related worsening of memory performance (Vakil \& Blachstein, 1997; Benedict et al. 1998), unrelated to the effect of 
cannabis exposure or the duration of exposure to cannabis. In the studies included in the present metaanalysis, healthy cannabis users and non-users did not differ in their average age, implicating that age was not a confounding factor in this group. However, in the studies that investigated patients with psychosis, cannabis-using patients were on average 5 years younger than the non-users (see Fig. 3). Performance in verbal memory tests are sensitive to changes in age of testing within the age ranges of participants reported in these studies (Hulicka, 1966), with younger age being associated with better performance in the Hopkins Verbal Learning Test (Benedict et al. 1998) as well as in learning and recall measures from the RAVLT (Vakil \& Blachstein, 1997). This may partly explain the better memory performance in cannabis-using patients relative to non-users.

A further explanation for the paradoxical findings in patients lies in the fact that many patients with schizophrenia are thought to have a neurodevelopmental disorder (Murray \& Lewis, 1987) while drug abuse may provide an alternative pathway to developing psychosis (Murray et al. 2013). Such a view would suggest that cannabis-using patients with psychosis may represent a subgroup with less neurodevelopmental pathology (Yücel et al. 2012; Ferraro et al. 2013) and less neurocognitive impairment than non-using patients in the first place. Perhaps, another explanation that does not pitch the neurodevelopmental pathway and drug abuse as alternative pathways to psychosis may be that cannabis-using patients with psychosis have better neurocognitive function to begin with. This idea is further supported by reports of absence of impaired cognition in association with use of a number of psychoactive substances (alcohol, cannabis, hallucinogens, cocaine, stimulants) in those with psychosis (Pencer \& Addington, 2003; Potvin et al. 2008) and evidence that substance-using schizophrenia patients were characterized by better social functioning and fewer negative symptoms when compared with non-users (Salyers \& Mueser, 2001; Carey et al. 2003).

Duration of abstinence from cannabis had a significant effect on memory function in both groups, such that the longer the duration of abstinence from cannabis, the smaller was the size of impairment observed, suggesting perhaps that worse memory performance associated with cannabis use may be largely but not completely reversible. These results are consistent with studies in healthy users reporting smaller effects of cannabis on memory in former users when compared with those who had never used (Fried et al. 2005) and reversible effects of cannabis on short-term memory function following abstinence in humans (Pope et al. 2001; Tait et al. 2011) and animals (Nakamura et al. 1991). Frequency of cannabis use also appeared to moderate the effects on memory in healthy individuals but not in patients consistent with evidence that the effects of cannabis are likely to depend on dose (Curran et al. 2002) and frequency of use (Fried et al. 2005). The evidence is less clear in this regard in patients with psychosis, with abstinence being associated with better performance in some (Rabin et al. 2012) but not all studies (Bugra et al. 2013). Similarly, an association between cognitive performance and duration and frequency of cannabis use was found in some studies including patients with psychosis (Wobrock et al. 2013), whilst others failed to show a correlation between duration of cannabis exposure and age at first cannabis use (Cunha et al. 2013).

An interesting trend in our analysis was the doubling of the effect on memory in healthy cannabis users reported in studies published after 2000 relative to those in the previous period (see Fig. 5). More speculatively, this may be related to the changing potency of cannabis that is used recreationally (Bowman \& Pihl, 1973; Soueif, 1976). In contrast to old-fashioned herbal cannabis and resin consumed in the 1970s and 1980s, for example comprising about 3-4\% THC (Poulsen \& Sutherland, 2000), THC content in currently available herbal cannabis has increased to about $8 \%$, and 'skunk' or sinsemilla is increasingly dominating the market in the UK and other European countries, a more potent variant containing about $16 \%$ THC (Hardwick \& King, 2008). However, in this context we cannot rule out the possibility that some of the between-study variation arose owing to changes in the quality of research conducted, i.e. that greater methodological rigor was introduced by studies published after 2000.

Certain limitations are to be considered when interpreting the results of the present meta-analysis. First, a causal relationship between cannabis use and memory impairments cannot be inferred given the crosssectional nature of the studies. Methodological heterogeneity of studies included is another important caveat inherent to the meta-analytic approach. In particular for the present meta-analysis, an obvious limitation is related to the use of different cognitive tests administered to measure the same memory dimension. Combining the results of different cognitive tests meta-analytically is an approach that has been employed before (Kambeitz et al. 2012) and sensitivity analysis indicates that this is unlikely to be an issue (see Supplementary Table S7). Conclusions regarding the impact of depression and differences in functioning between the patients $v$. healthy samples and cannabis user $v$. non-user comparison groups are of limited generalizability, given the diversity of measures administered to measure level of functioning, depression and pre-morbid IQ (see Supplementary Table S2). 
Furthermore, they have been administered by raters with different backgrounds (e.g. clinically trained $v$. non-trained researchers $v$. self-ratings by subjects) in different contexts (e.g. psychiatric services $v$. research facilities). Another important limitation relates to heterogeneity in the criteria used to define the user $v$. nonuser subgroups in the studies (see Supplementary Table S9). This was less of a problem in studies involving healthy individuals, with only one study defining non-users as those who did not use cannabis in the previous year (Wadsworth et al. 2006) and one defining non-users as those who were not diagnosed with cannabis use disorder at the assessment (Smith et al. 2014), whilst the remaining studies defined non-users as those who never used cannabis in their life or never used the substance regularly. Caution is also warranted in interpreting the present data regarding the moderating effects of cannabis use patterns such as abstinence, frequency, duration and age of onset of use, as they were mainly based on self-reports and hence represent only crude approximations of actual exposures. Effects of these moderators were investigated independently, rather than within multifactorial prediction models, which was not possible in this meta-analysis as only a few studies reported data on all parameters of cannabis use. However, this limits the interpretation of these results, given that these moderators interact with each other to influence memory (Wagner et al. 2010) and the results may be susceptible to bias considering that only a subset of studies was available for moderator analysis (see Supplementary Table S10). It is also worth noting that various factors may underlie motivation to use cannabis among patients with psychosis, including using it as a form of self-medication. Consistent with this idea, it may be possible that some patients may have used cannabis to treat their pre-existing cognitive deficits. However, this aspect was not addressed in the present paper as the focus of this meta-analysis was not to investigate the reasons as to why patients with psychosis use cannabis, but to estimate the magnitude of the effects of cannabis on memory, irrespective of the reasons for its use. Generalizability of the results may also be limited to Europe, North America and Australia, as only a few studies came from Asia or Africa.

\section{Conclusions}

These results confirm that regular cannabis use impairs memory function across a range of different memory domains, at least in healthy individuals. A similar adverse effect was not observed in patients with psychosis, which may reflect the possibility that cannabis users among patients with psychosis comprise a less depressed, less developmentally impaired, and younger subgroup in comparison with non-using patients. Duration of abstinence from cannabis appeared to reduce the effects on memory in both healthy individuals and patients with a psychotic disorder, underlining the importance of harm-reduction strategies. Our findings highlight a need for future studies employing longitudinal design in adequately matched participant groups on potential confounding factors, to definitively establish the effects of regular use of the world's most widely used illicit drug on memory function.

\section{Supplementary material}

For supplementary material accompanying this paper visit http://dx.doi.org/10.1017/S0033291715001646

\section{Acknowledgements}

S.B. has received support from the National Institute of Health Research (NIHR), UK (NIHR Clinician Scientist Award; NIHR CS-11-001) and the UK Medical Research Council (MR/J012149/1) as well as from the NIHR Mental Health Biomedical Research Centre at South London and Maudsley NHS Foundation Trust and King's College London.

We would like to acknowledge Professor Stefan Leucht who commented on an earlier draft of the paper. We thank all the authors of the included studies, especially Ruud van Winkel, M.D., M.Sc., Ph.D. and Carissa Coulston, M.Sc., Ph.D. for kindly sharing their unpublished data for inclusion in this meta-analysis.

\section{Declaration of Interest}

None.

\section{References}

Bahorik AL, Newhill CE, Eack SM (2013). Neurocognitive functioning of individuals with schizophrenia: using and not using drugs. Schizophrenia Bulletin 40, 856-867.

Beller EM, Glasziou PP, Altman DG, Hopewell S, Bastian H, Chalmers I, Gøtzsche PC, Lasserson T, Tovey D (2013). PRISMA for abstracts: reporting systematic reviews in journal and conference abstracts. PLoS Medicine 10, e1001419.

Benedict RHB, Schretlen D, Groninger L, Brandt J (1998). Hopkins Verbal Learning Test-revised: normative data and analysis of inter-form and test-retest reliability. Clinical Neuropsychologist 12, 43-55.

Bhattacharyya S, Atakan Z, Martin-Santos R, Crippa JA, Kambeitz J, Prata D, Williams S, Brammer M, Collier DA, McGuire PK (2012). Preliminary report of biological basis of sensitivity to the effects of cannabis on psychosis: AKT1 and DAT1 genotype modulates the effects of 
$\delta$-9-tetrahydrocannabinol on midbrain and striatal function. Molecular Psychiatry 17, 1152-1155.

Bhattacharyya S, Fusar-Poli P, Borgwardt S, Martin-Santos R, Nosarti C, O'Carroll C, Allen P, Seal ML, Fletcher PC, Crippa JA, Giampietro V, Mechelli A, Atakan Z, McGuire P (2009). Modulation of mediotemporal and ventrostriatal function in humans by $\Delta 9$-tetrahydrocannabinol: a neural basis for the effects of Cannabis sativa on learning and psychosis. Archives of General Psychiatry 66, 442-451.

Bolla KI, Brown K, Eldreth D, Tate K, Cadet JL (2002). Doserelated neurocognitive effects of marijuana use. Neurology 59, 1337-1343.

Borenstein M, Hedges LV, Higgins JP, Rothstein HR (2011). Introduction to Meta-Analysis. Wiley: Chichester.

Bowman M, Pihl RO (1973). Cannabis: psychological effects of chronic heavy use. Psychopharmacologia 29, 159-170.

Bugra H, Studerus E, Rapp C, Tamagni C, Aston J, Borgwardt S, Riecher-Rössler A (2013). Cannabis use and cognitive functions in at-risk mental state and first episode psychosis. Psychopharmacology (Berlin) 230, 299-308.

Burt DB, Zembar MJ, Niederehe G (1995). Depression and memory impairment: a meta-analysis of the association, its pattern, and specificity. Psychological Bulletin 117, 285-305.

Carey KB, Carey MP, Simons JS (2003). Correlates of substance use disorder among psychiatric outpatients: focus on cognition, social role functioning and psychiatric status. Journal of Nervous and Mental Disease 191, 300-308.

Cunha PJ, Rosa PGP, Ayres Ade M, Duran FLS, Santos LC, Scazufca M, Menezes PR, dos Santos B, Murray RM, Crippa JAS, Busatto GF, Schaufelberger MS (2013). Cannabis use, cognition and brain structure in first-episode psychosis. Schizophrenia Research 147, 209-215.

Curran VH, Brignell C, Fletcher S, Middleton P, Henry J (2002). Cognitive and subjective dose-response effects of acute oral $\Delta$-tetrahydrocannabinol (THC) in infrequent cannabis users. Psychopharmacology (Berlin) 164, 61-70.

D'Souza DC, Abi-Saab WM, Madonick S, Forselius-Bielen K, Doersch A, Braley G, Gueorguieva R, Cooper TB, Krystal JH (2005). $\Delta$-9-Tetrahydrocannabinol effects in schizophrenia: implications for cognition, psychosis, and addiction. Biological Psychiatry 57, 594-608.

D'Souza DC, Perry E, MacDougall L, Ammerman Y, Cooper T, Wu YT, Braley G, Gueorguieva R, Krystal JH (2004). The psychotomimetic effects of intravenous $\Delta$-9-tetrahydrocannabinol in healthy individuals: implications for psychosis. Neuropsychopharmacology 29, 1558-1572.

Donoghue K, Doody GA (2012). Effect of illegal substance use on cognitive function in individuals with a psychotic disorder, a review and meta-analysis. Neuropsychology 26, 785-801.

Donoghue K, Mazzoncini R, Hart J, Zanelli J, Morgan C, Dazzan P, Morgan KD, Murray RM, Jones PB, Doody GA (2012). The differential effect of illicit drug use on cognitive function in first-episode psychosis and healthy controls. Acta Psychiatrica Scandinavica 125, 400-411.

Eichenbaum H (1993). Memory, Amnesia, and the Hippocampal System. The MIT Press: Cambridge, MA.
Englund A, Morrison PD, Nottage J, Hague D, Kane F, Bonaccorso S, Stone JM, Reichenberg A, Brenneisen R, Holt D (2013). Cannabidiol inhibits THC-elicited paranoid symptoms and hippocampal-dependent memory impairment. Journal of Psychopharmacology 27, 19-27.

Ferraro L, Russo M, O'Connor J, Wiffen BDR, Falcone MA, Sideli L, Gardner-Sood P, Stilo S, Trotta A, Dazzan P, Mondelli V, Taylor H, Friedman B, Sallis H, La Cascia C, La Barbera D, David AS, Reichenberg A, Murray R, Di Forti M (2013). Cannabis users have higher premorbid IQ than other patients with first onset psychosis. Schizophrenia Research 150, 129-135.

Fletcher PC, Honey GD (2006). Schizophrenia, ketamine and cannabis: evidence of overlapping memory deficits. Trends in Cognitive Sciences 10, 167-174.

Fried PA, Watkinson B, Gray R (2005). Neurocognitive consequences of marihuana - a comparison with pre-drug performance. Neurotoxicology and Teratology 27, 231-239.

Gonzalez R, Carey C, Grant I (2002). Nonacute (residual) neuropsychological effects of cannabis use: a qualitative analysis and systematic review. Journal of Clinical Pharmacology 42 (11 Suppl.), 48S-57S.

Grant I, Gonzales R, Carey CL, Natarajan L, Wolfson T (2003). Non-acute (residual) neurocognitive effects of cannabis use: a meta-analytic study. Journal of the International Neuropsychological Society 9, 679-689.

Gruber SA, Sagar KA, Dahlgren MK, Racine M, Lukas SE (2012). Age of onset of marijuana use and executive function. Psychology of Addictive Behaviors 26, 496-506.

Hardwick S, King LA (2008). Home Office Cannabis Potency Study 2008. United Kingdom: Home Office Scientific Development Branch: St Albans.

Herkenham M, Lynn AB, Little MD, Johnson MR, Melvin LS, De Costa BR, Rice KC (1990). Cannabinoid receptor localization in brain. Proceedings of the National Academy of Sciences of the USA 87, 1932-1936.

Higgins JP, Green S (editors) (2008). Cochrane Handbook for Systematic Reviews of Interventions. Wiley Online Library: Chichester.

Hulicka IM (1966). Age differences in Wechsler Memory Scale scores. Journal of Genetic Psychology 109, 135-145.

Iversen L (2003). Cannabis and the brain. Brain 126, 1252 1270.

Jager G, Kahn RS, Van Den Brink W, Van Ree JM, Ramsey NF (2006). Long-term effects of frequent cannabis use on working memory and attention: an fMRI study. Psychopharmacology (Berlin) 185, 358-368.

Jockers-Scherubl MC, Wolf T, Radzei N, Schlattmann P, Rentzsch J, Gomez-Carrillo de Castro A, Kuhl KP (2007). Cannabis induces different cognitive changes in schizophrenic patients and in healthy controls. Progress in Neuro-Psychopharmacology and Biological Psychiatry 31, 1054-1063.

Kambeitz JP, Bhattacharyya S, Kambeitz-Ilankovic LM, Valli I, Collier DA, McGuire P (2012). Effect of BDNF val $^{66}$ met polymorphism on declarative memory and its neural substrate: a meta-analysis. Neuroscience and Biobehavioral Reviews 36, 2165-2177. 
Kanayama G, Rogowska J, Pope HG, Gruber SA, Yurgelun-Todd DA (2004). Spatial working memory in heavy cannabis users: a functional magnetic resonance imaging study. Psychopharmacology (Berlin) 176, 239-247.

Korver N, Nieman DH, Becker HE, van de Fliert JR, Dingemans PH, de Haan L, Spiering M, Schmitz N, Linszen DH (2010). Symptomatology and neuropsychological functioning in cannabis using subjects at ultra-high risk for developing psychosis and healthy controls. Australian and New Zealand Journal of Psychiatry 44, 230-236.

Koskinen J, Löhönen J, Koponen H, Isohanni M, Miettunen J (2010). Rate of cannabis use disorders in clinical samples of patients with schizophrenia: a meta-analysis.

Schizophrenia Bulletin 36, 1115-1130.

Lane SD, Cherek DR, Lieving LM, Tcheremissine OV (2005). Marijuana effects on human forgetting functions. Journal of the Experimental Analysis of Behavior 83, 67-83.

Leeson VC, Harrison I, Ron MA, Barnes TR, Joyce EM (2012). The effect of cannabis use and cognitive reserve on age at onset and psychosis outcomes in first-episode schizophrenia. Schizophrenia Bulletin 38, 873-880.

Linszen DH, Dingemans PM, Nugter MA, Van der Does AJ, Scholte WF, Lenior MA (1997). Patient attributes and expressed emotion as risk factors for psychotic relapse. Schizophrenia Bulletin 23, 119-130.

Løberg E-M, Hugdahl K (2009). Cannabis use and cognition in schizophrenia. Frontiers in Human Neuroscience 3, 53.

Løberg EM, Hugdahl K, Jørgensen HA (2008). Lower neurocognitive vulnerability in schizophrenia with a history of cannabis abuse? Schizophrenia Research 98, 73.

Lutgens D, Lepage M, Iyer S, Malla A (2014). Predictors of cognition in first episode psychosis. Schizophrenia Research 152, 164-169.

Matochik JA, Eldreth DA, Cadet JL, Bolla KI (2005). Altered brain tissue composition in heavy marijuana users. Drug and Alcohol Dependence 77, 23-30.

McDermott LM, Ebmeier KP (2009). A meta-analysis of depression severity and cognitive function. Journal of Affective Disorders 119, 1-8.

Meier MH, Caspi A, Ambler A, Harrington H, Houts R, Keefe RS, McDonald K, Ward A, Poulton R, Moffitt TE (2012). Persistent cannabis users show neuropsychological decline from childhood to midlife. Proceedings of the National Academy of Sciences of the USA 109, E2657-E2664.

Miles H, Johnson S, Amponsah-Afuwape S, Finch E, Leese M, Thornicroft G (2003). Characteristics of subgroups of individuals with psychotic illness and a comorbid substance use disorder. Psychiatric Services 54, 554-561.

Moreno-Granados JM, Ferrín M, Salcedo-Marín DM, Ruiz-Veguilla M (2014). Neuropsychological assessment of memory in child and adolescent first episode psychosis: cannabis and the «paradox effect». Revista Brasileira de Psiquiatria 7, 13-24.

Morrison PD, Zois V, McKeown DA, Lee TD, Holt DW, Powell JF, Kapur S, Murray RM (2009). The acute effects of synthetic intravenous $\Delta$ 9-tetrahydrocannabinol on psychosis, mood and cognitive functioning. Psychological Medicine 39, 1607-1616.
Moss HB, Chen CM, Yi HY (2013). Early adolescent patterns of alcohol, cigarettes, and marijuana polysubstance use and young adult substance use outcomes in a nationally representative sample. Drug and Alcohol Dependence 136, 51-62.

Murray RM, Lewis SW (1987). Is schizophrenia a neurodevelopmental disorder? British Medical Journal (Clinical Research ed.) 295, 681-682.

Murray RM, Paparelli A, Morrison PD, Marconi A, Di Forti M (2013). What can we learn about schizophrenia from studying the human model, drug-induced psychosis? American Journal of Medical Genetics Part B: Neuropsychiatric Genetics 162B, 661-670.

Nakamura EM, Da Silva EA, Concilio GV, Adrian Wilkinson D, Masur J (1991). Reversible effects of acute and long-term administration of $\Delta-9$-tetrahydrocannabinol (THC) on memory in the rat. Drug and Alcohol Dependence 28, 167-175.

Packard MG, Knowlton BJ (2002). Learning and memory functions of the basal ganglia. Annual Review of Neuroscience 25, 563-593.

Pencer A, Addington J (2003). Substance use and cognition in early psychosis. Journal of Psychiatry and Neuroscience 28, 48-54.

Penk WE, Flannery RB Jr, Irvin E, Geller J, Fisher W, Hanson MA (2000). Characteristics of substance-abusing persons with schizophrenia: the paradox of the dually diagnosed. Journal of Addictive Diseases 19, 23-30.

Pertwee RG (2008). Ligands that target cannabinoid receptors in the brain: from THC to anandamide and beyond. Addiction Biology 13, 147-159.

Pezdek K, Evans GW (1979). Visual and verbal memory for objects and their spatial locations. Journal of Experimental Psychology Human Learning and Memory 5, 360-373.

Pope HG, Gruber AJ, Hudson JI, Cohane G, Huestis MA, Yurgelun-Todd D (2003). Early-onset cannabis use and cognitive deficits: what is the nature of the association? Drug and Alcohol Dependence 69, 303-310.

Pope HG, Gruber AJ, Hudson JI, Huestis MA, YurgelunTodd D (2001). Neuropsychological performance in longterm cannabis users. Archives of General Psychiatry 58, 909-915.

Potvin S, Joyal CC, Pelletier J, Stip E (2008). Contradictory cognitive capacities among substance-abusing patients with schizophrenia: a meta-analysis. Schizophrenia Research 100, 242-251.

Poulsen HA, Sutherland GJ (2000). The potency of cannabis in New Zealand from 1976 to 1996. Science and Justice 40, 171-176.

Rabin RA, Zakzanis KK, Daskalakis ZJ, George TP (2012). Effects of cannabis use status on cognitive function, in males with schizophrenia. Psychiatry Research 206, 158-165.

Rabin RA, Zakzanis KK, George TP (2011). The effects of cannabis use on neurocognition in schizophrenia: a meta-analysis. Schizophrenia Research 128, 111-116.

Ranganathan M, D'Souza DC (2006). The acute effects of cannabinoids on memory in humans: a review. Psychopharmacology 188, 425-444.

Ruiz-Veguilla M, Callado LF, Ferrin M (2012). Neurological soft signs in patients with psychosis and cannabis abuse: a 
systematic review and meta-analysis of paradox. Current Pharmaceutical Design 18, 5156-5164.

Salyers MP, Mueser KT (2001). Social functioning, psychopathology, and medication side effects in relation to substance use and abuse in schizophrenia. Schizophrenia Research 48, 109-123.

Schnell T, Koethe D, Daumann J, Gouzoulis-Mayfrank E (2009). The role of cannabis in cognitive functioning of patients with schizophrenia. Psychopharmacology (Berlin) 205, 45-52.

Schoeler T, Bhattacharyya S (2013). The effect of cannabis use on memory function: an update. Journal of Substance Abuse and Rehabilitation 4, 11-27.

Schreiner AM, Dunn ME (2012). Residual effects of cannabis use on neurocognitive performance after prolonged abstinence: a meta-analysis. Experimental and Clinical Psychopharmacology 20, 409-420.

Smith K, Flatley J (2011). Drug misuse declared: findings from the 2010/11 British Crime Survey England and Wales. Home Office Statistical Bulletin 12/11 (https://www.gov.uk/ government/uploads/system/uploads/attachment_data/file/ 116333/hosb1211.pdf). Accessed August 2015.

Smith MJ, Cobia DJ, Wang L, Alpert KI, Cronenwett WJ, Goldman MB, Mamah D, Barch DM, Breiter HC, Csernansky JG (2014). Cannabis-related working memory deficits and associated subcortical morphological differences in healthy individuals and schizophrenia subjects. Schizophrenia Bulletin 40, 287-299.

Sneider JT, Pope HG Jr, Silveri MM, Simpson NS, Gruber SA, Yurgelun-Todd DA (2008). Differences in regional blood volume during a 28-day period of abstinence in chronic cannabis smokers. European Neuropsychopharmacology 18, 612-619.

Solowij N, Battisti R (2008). The chronic effects of cannabis on memory in humans: a review. Current Drug Abuse Reviews 1, 81-98.

Solowij N, Pesa N (2010). Cognitive abnormalities and cannabis use. Revista Brasileira de Psiquiatria 32 (Suppl. 1), S31-S40.

Song F, Sheldon TA, Sutton AJ, Abrams KR, Jones DR (2001). Methods for exploring heterogeneity in metaanalysis. Evaluation and the Health Professions 24, 126-151.
Soueif MI (1976). Differential association between chronic cannabis use and brain function deficits. Annals of the New York Academy of Sciences 282, 323-343.

Tait RJ, Mackinnon A, Christensen H (2011). Cannabis use and cognitive function: 8-year trajectory in a young adult cohort. Addiction 106, 2195-2203.

UN Office on Drugs and Crime (2010). World Drug Report 2010. United Nations Publications: New York.

Vakil E, Blachstein H (1997). Rey AVLT: developmental norms for adults and the sensitivity of different memory measures to age. Clinical Neuropsychologist 11, 356-369.

Viechtbauer W (2010). Conducting meta-analyses in R with the metafor package. Journal of Statistical Software 36, $1-48$.

Wadsworth EJK, Moss SC, Simpson SA, Smith AP (2006). Cannabis use, cognitive performance and mood in a sample of workers. Journal of Psychopharmacology 20, 14-23.

Wagner D, Becker B, Gouzoulis-Mayfrank E, Daumann J (2010). Interactions between specific parameters of cannabis use and verbal memory. Progress in Neuro-Psychopharmacology and Biological Psychiatry 34, 871-876.

Wobrock T, Falkai P, Schneider-Axmann T, Hasan A, Galderisi S, Davidson M, Kahn RS, Derks EM, Boter H, Rybakowski JK (2013). Comorbid substance abuse in firstepisode schizophrenia: effects on cognition and psychopathology in the EUFEST study. Schizophrenia Research 147, 132-139.

Wojtalik JA, Barch DM (2014). An fMRI study of the influence of a history of substance abuse on working memory-related brain activation in schizophrenia. Frontiers in Psychiatry 5, 1.

Yücel M, Bora E, Lubman DI, Solowij N, Brewer WJ, Cotton SM, Conus P, Takagi MJ, Fornito A, Wood SJ (2012). The impact of cannabis use on cognitive functioning in patients with schizophrenia: a meta-analysis of existing findings and new data in a first-episode sample. Schizophrenia Bulletin 38, 316-330.

Yucel M, Solowij N, Respondek C, Whittle S, Fornito A, Pantelis C, Lubman DI (2008). Regional brain abnormalities associated with long-term heavy cannabis use. Archives of General Psychiatry 65, 694-701. 